\title{
Super-weak force
}

\section{Zoltán Trócsányi*†}

Institute for Theoretical Physics, ELTE Eötvös Loránd University,

Pázmány Péter sétány 1/A, H-1117 Budapest, Hungary

and MTA-DE Particle Physics Research Group, H-4010 Debrecen, PO Box 105, Hungary

E-mail: Zoltan.Trocsanyi@cern.ch

We summarize the current status of particle physics, collecting the established deviations from the standard model of particle interactions both at the energy and the intensity frontier as well as in cosmology. We propose a specific $U(1)$ extension of the standard model gauge group of particle interactions and discuss the possible consequences of the model concerning the observed deviations.

International Conference on Precision Physics and Fundamental Physical Constants - FFK2019 9-14 June, 2019

Tihany, Hungary

* Speaker.

${ }^{\dagger}$ Work supported by grant K 125105 of the National Research, Development and Innovation Fund in Hungary. 


\section{Introduction}

At this workshop three dedicated talks discussed the status of particle physics from measurements preformed at high energy collider experiments of the LHC, focusing mostly on the experimental status of the standard model $[1,2,3]$. The conclusions can be summarized easily. Firstly, the LHC has become a precision machine where the uncertainties of individual measurements are approaching those of previous measurements combined (obtained by the experiments of the Large Electron-Positron collider, the Stanford Linear Collider and Tevatron). Secondly, the results are all compatible with the predictions of the standard model. There was also a talk on searches for signs of new physics at the LHC [4] where we learnt that so far only exclusion limits were found, no new particles.

The healthy status of the standard model and the fruitless search for new particles might hint that the standard model is the complete theory of particle interactions. Yet there are several confirmed signs of new physics that call clearly for the extension of the standard model: we know that (i) the universe at large scales is described precisely by the cosmological standard model that uses the existence of cold dark matter, contributing to about $25 \%$ of the energy density of the universe; (ii) neutrino flavours oscillate, which is possible only if they have masses; (iii) we exist, meaning that there is a baryon-anti-baryon asymmetry in the universe, whose magnitude cannot be explained by the violation of CP symmetry in the standard model [5]. (iv) Using the known parameters of the standard model, the quartic coupling in the Brout-Englert-Higgs (BEH) potential becomes negative at around $10^{11} \mathrm{GeV}[6,7]$, suggesting potential instability of the early universe. While the estimates for the tunneling time of the universe into a more stable ground state give longer time than the age of the universe since the Big Bang, meaning that the universe is in a metastable state, such a potential instability could be fatal at early times when the characteristic energy scale of particle interactions was higher than the scale of instability. (v) Signs of inflation are hinted by structure of the power spectrum of the cosmic microwave background radiation and the accelerated expansion of the present universe has been observed [5]. According to the current consensus, these observations should be explained consistently by an extension of the standard model.

In the present contribution we propose such an extension. There have been many attempts in the literature to devise models beyond the standard one. As the effect of the extension must be very small on the measurements at past and present high energy colliders, the new physics has to be suppressed in the region of interaction energies studied so far. Such a suppression can be achieved if the masses of new states are so large that they cannot be excited at the LHC, or the couplings of the new forces are very small. Most of the studies on beyond standard model assume the first option, with the hope that perhaps the masses of new particles are within the reach of the LHC, as one may expect for instance in supersymmetric extensions. Here we describe an extension of the standard model along the same underlying principles as the standard model is built, and propose to constrain the parameter space by confrontation with existing results, which points towards the case of small couplings.

\section{Definition of the model}

The standard model of particle interactions is based on the concepts of local gauge invariance 
against the elements of the standard model gauge group $G_{\mathrm{SM}}=S U(3)_{\mathrm{c}} \otimes S U(2)_{\mathrm{L}} \otimes U(1)_{Y}[8]$ and spontaneous symmetry breaking $[9,10]$. The particle charges ensure that the model is free from gauge and gravity anomalies. It is a highly efficient model, with the only exception of economical description is the relatively large number of Yukawa couplings of the fermions needed to explain their non-vanishing masses. Yet the generation of the fermion masses is also efficient in the sense that it uses the same spontaneous symmetry breaking of the scalar field to which all other particles owe their masses. In this spirit, it is reasonable to expect that the masses of the neutrinos should be explained by Yukawa couplings, too, which requires the existence of right-handed neutrinos, so we enlarge the fermion sector and introduce the notation

$$
\begin{array}{ll}
\psi_{q, 1}^{f}=\left(\begin{array}{c}
U^{f} \\
D^{f}
\end{array}\right)_{\mathrm{L}} \quad \psi_{q, 2}^{f}=U_{\mathrm{R}}^{f}, \quad \psi_{q, 3}^{f}=D_{\mathrm{R}}^{f} \\
\psi_{l, 1}^{f}=\left(\begin{array}{c}
v^{f} \\
\ell^{f}
\end{array}\right)_{\mathrm{L}} \quad \psi_{l, 2}^{f}=v_{\mathrm{R}}^{f}, \quad \psi_{l, 3}^{f}=\ell_{\mathrm{R}}^{f}
\end{array}
$$

for the chiral quark fields $\psi_{q}$ and for the chiral lepton fields $\psi_{l}$. In Eq. (2.1) L and R denote the left and right-handed projections of the same field,

$$
\psi_{\mathrm{L} / \mathrm{R}} \equiv \psi_{\mp}=\frac{1}{2}\left(1 \mp \gamma_{5}\right) \psi \equiv P_{\mathrm{L} / \mathrm{R}} \psi
$$

If the underlying gauge group is $G_{\mathrm{SM}}$ only, then these neutrinos do not have any other interactions as anomaly cancellation requires them being singlet, neutral states under $G_{\mathrm{SM}}$ transformations. In order to make these neutrinos charged, we need an extra gauge group. Thus we extend $G_{\mathrm{SM}}$ to $G_{\mathrm{SM}} \otimes U(1)_{Z}$ as a simplest option. The requirement of local gauge invariance of the Dirac Lagrangian

$$
\mathscr{L}_{\mathrm{D}}=\mathrm{i} \sum_{f=1}^{3} \sum_{j=1}^{3}\left(\bar{\psi}_{q, j}^{f}(x) \not \phi_{j} \psi_{q, j}^{f}(x)+\bar{\psi}_{l, j}^{f}(x) \not \phi_{j} \psi_{l, j}^{f}(x)\right)
$$

under the extended gauge group leads to the covariant derivatives

$$
D_{j}^{\mu}=\partial^{\mu}+\mathrm{i} g_{\mathrm{L}} \boldsymbol{T} \cdot \boldsymbol{W}^{\mu}+\mathrm{i} g_{Y} y_{j} B^{\prime \mu}+\mathrm{i}\left(g_{Z}^{\prime} z_{j}-g_{Y}^{\prime} y_{j}\right) Z^{\prime \mu}
$$

where the primes on the vector fields and gauge couplings indicates that the kinetic mixing between the $U(1)$ fields have been taken into account [11]. The matrices $\boldsymbol{T}=\left(T_{1}, T_{2}, T_{3}\right)$ are $\frac{1}{2}$ times the Pauli matrices, $y_{j}$ is the hypercharge, while $z_{j}$ denotes the $Z$-charge of the field $\psi_{j}$. There is a lot of freedom how to choose the $Z$-charges. Here we assume that the charges do not depend on the families and the theory is free from gauge and gravity anomalies, just as in the standard model. With this assumption the assignment for the $Z$-charges of the fermions can be expressed using two free numbers $Z_{1}$ and $Z_{2}$ of the $U$ quark fields. The rest of the charges must take values as given in Table 1 [12]. We specify the two numbers $Z_{1}$ and $Z_{2}$ below.

For the right-handed neutrinos renormalizable, gauge invariant Yukawa interactions are also possible in Majorana form if we allow for the existence of a new complex scalar field $\chi$ that transforms as a singlet under $G_{\mathrm{SM}}$ transformations. Then the gauge invariant Lagrangian of the scalar fields is

$$
\mathscr{L}_{\phi, \chi}=\left[D_{\phi \mu} \phi\right]^{*} D_{\phi}^{\mu} \phi+\left[D_{\chi \mu} \chi\right]^{*} D_{\chi}^{\mu} \chi-V(\phi, \chi)
$$


Table 1: Assignments for the representations (for $S U(N)$ ) and charges (for $U(1)$ ) of fermion and scalar fields of the complete model. The charges $y_{j}$ denote the eigenvalue of $Y / 2$, with $Y$ being the hypercharge operator and $z_{j}$ denote the supercharges of the fields $\psi_{j}$ of Eq. $(2.1)(j=1,2,3)$. The right-handed Dirac neutrinos $v_{\mathrm{R}}$ are sterile under the $G_{\mathrm{SM}}$ group. The sixth column gives a particular realization of the $U(1)_{Z}$ charges, motivated below, and the last one is added for later convenience.

\begin{tabular}{lccrr|rc}
\hline \hline field & $S U(3)_{\mathrm{c}}$ & $S U(2)_{\mathrm{L}}$ & $y_{j}$ & $z_{j}$ & $z_{j}$ & $r_{j}=z_{j} / z_{\phi}-y_{j}$ \\
\hline$U_{\mathrm{L}}, D_{\mathrm{L}}$ & 3 & 2 & $\frac{1}{6}$ & $Z_{1}$ & $\frac{1}{6}$ & 0 \\
$U_{\mathrm{R}}$ & 3 & 1 & $\frac{2}{3}$ & $Z_{2}$ & $\frac{7}{6}$ & $\frac{1}{2}$ \\
$D_{\mathrm{R}}$ & 3 & 1 & $-\frac{1}{3}$ & $2 Z_{1}-Z_{2}$ & $-\frac{5}{6}$ & $-\frac{1}{2}$ \\
$v_{\mathrm{L}}, \ell_{\mathrm{L}}$ & 1 & 2 & $-\frac{1}{2}$ & $-3 Z_{1}$ & $-\frac{1}{2}$ & 0 \\
$v_{\mathrm{R}}$ & 1 & 1 & 0 & $Z_{2}-4 Z_{1}$ & $\frac{1}{2}$ & $\frac{1}{2}$ \\
$\ell_{\mathrm{R}}$ & 1 & 1 & -1 & $-2 Z_{1}-Z_{2}$ & $-\frac{3}{2}$ & $-\frac{1}{2}$ \\
$\phi$ & 1 & 2 & $\frac{1}{2}$ & $z_{\phi}$ & 1 & $\frac{1}{2}$ \\
$\chi$ & 1 & 1 & 0 & $z_{\chi}$ & -1 & -1 \\
\hline \hline
\end{tabular}

where the covariant derivative for the scalar $s(s=\phi, \chi)$ is

$$
D_{s}^{\mu}=\partial^{\mu}+\mathrm{i} g_{\mathrm{L}} \boldsymbol{T} \cdot \boldsymbol{W}^{\mu}+\mathrm{i} g_{Y} y_{s} B^{\prime \mu}+\mathrm{i}\left(g_{Z}^{\prime} z_{s}-g_{Y}^{\prime} y_{s}\right) Z^{\prime \mu}
$$

and the potential energy contains also a mixed coupling term $-\lambda|\phi|^{2}|\chi|^{2}$ of the scalar fields in the Lagrangian:

$$
V(\phi, \chi)=V_{0}-\mu_{\phi}^{2}|\phi|^{2}-\mu_{\chi}^{2}|\chi|^{2}+\left(|\phi|^{2},|\chi|^{2}\right)\left(\begin{array}{cc}
\lambda_{\phi} & \frac{\lambda}{2} \\
\frac{\lambda}{2} & \lambda_{\chi}
\end{array}\right)\left(\begin{array}{c}
|\phi|^{2} \\
|\chi|^{2}
\end{array}\right) .
$$

After spontaneous symmetry breaking, $G \rightarrow S U(3)_{\mathrm{c}} \otimes U(1)_{Q}$, we parametrize the scalar fields as

$$
\phi=\frac{1}{\sqrt{2}} \mathrm{e}^{\mathrm{i} \boldsymbol{T} \cdot \boldsymbol{\xi}(x) / v}\left(\begin{array}{c}
0 \\
v+h^{\prime}(x)
\end{array}\right) \text { and } \chi(x)=\frac{1}{\sqrt{2}} \mathrm{e}^{\mathrm{i} \eta(x) / w}\left(w+s^{\prime}(x)\right) .
$$

Then similarly as in the standard model, we can use the gauge invariance to choose the unitary gauge when

$$
\phi^{\prime}(x)=\frac{1}{\sqrt{2}}\left(\begin{array}{c}
0 \\
v+h^{\prime}(x)
\end{array}\right) \quad \text { and } \quad \chi^{\prime}(x)=\frac{1}{\sqrt{2}}\left(w+s^{\prime}(x)\right) .
$$

The fermion masses emerge from the Yukawa Lagrangian

$$
\mathscr{L}_{\mathrm{Y}}=-\left[c_{D} \bar{Q}_{\mathrm{L}} \cdot \phi D_{\mathrm{R}}+c_{U} \bar{Q}_{\mathrm{L}} \cdot \tilde{\phi} U_{\mathrm{R}}+c_{\ell} \bar{L}_{\mathrm{L}} \cdot \phi \ell_{\mathrm{R}}+c_{v} \bar{L}_{\mathrm{L}} \cdot \tilde{\phi} v_{\mathrm{R}}+\frac{1}{2} c_{\mathrm{R}} \overline{v_{\mathrm{R}}^{c}} v_{\mathrm{R}} \chi\right]+\text { h.c. }
$$

where the dot product abbreviates scalar products of $S U(2)$ doublets:

$$
\bar{Q}_{\mathrm{L}} \cdot \phi \equiv(\bar{U}, \bar{D})_{\mathrm{L}}\left(\begin{array}{c}
\phi^{(+)} \\
\phi^{(0)}
\end{array}\right), \quad \bar{Q}_{\mathrm{L}} \cdot \tilde{\phi} \equiv(\bar{U}, \bar{D})_{\mathrm{L}}\left(\begin{array}{r}
\phi^{(0) *} \\
-\phi^{(+) *}
\end{array}\right)
$$


$\bar{L} \equiv\left(\bar{v}_{\ell}, \bar{\ell}\right), c$ denotes charge conjugate of the field, $v^{c}=-\mathrm{i} \gamma_{2} v^{*}$ and h.c. means hermitian conjugate terms. The Yukawa couplings $c_{D}, c_{U}, c_{\ell}, c_{v}, c_{\mathrm{R}}$ are matrices in family indices and summation over the families is understood. The Majorana term of Eq. (2.10) (the last piece in the bracket) is gauge invariant if the $Z$-charge of the right-handed neutrinos and the new scalar are related by $z_{\chi}=-2 z_{v_{\mathrm{R}}}$. Here we fix the $Z$-charges of the left- and right-handed neutrinos to have opposite charges, so $Z_{2}-4 Z_{1}=3 Z_{1}$, solved by $Z_{1}=Z_{2} / 7$. We can set the overall scale of the $Z$-charges freely (it influences only on the value of the gauge coupling $g_{Z}^{\prime}$ ), and we choose $Z_{2}=7 / 6 \mathrm{im}$ plying $Z_{1}=1 / 6$. Then the $Z$-charge of the BEH scalar is $z_{\phi}=1$, while that of the new scalar is $z_{\chi}=-1=-z_{\phi}$. These charge assignments are shown in the sixth column of Table 1 explicitly.

We can re-parametrize the couplings to $Z^{\prime}$ using the new coupling

$$
g_{Z Y}^{\prime}=g_{Z}^{\prime}-g_{Y}^{\prime} .
$$

Then the covariant derivative in Eq. (2.4) becomes

$$
D_{j}^{\mu}=\partial^{\mu}+\mathrm{i} g_{\mathrm{L}} \boldsymbol{T} \cdot \boldsymbol{W}^{\mu}+\mathrm{i} y_{j} g_{Y} B^{\prime \mu}+\mathrm{i}\left(r_{j} g_{Z}^{\prime}+y_{j} g_{Z Y}^{\prime}\right) Z^{\prime \mu}
$$

where $r_{j}=z_{j}-y_{j}$ and its values are given explicitly in the last column of Table 1 . We see that the left-handed fields couple to the new gauge boson with their hypercharges only, while the right charges $r_{j}$ take opposite values for the right-handed up $\left(r_{j}=1 / 2\right)$ and down-type $\left(r_{j}=-1 / 2\right)$ fields.

After the spontaneous symmetry breaking of the vacuum of the scalar fields Eq. (2.10) leads to mass terms for the neutrinos:

$$
\mathscr{L}_{\mathrm{Y}}^{v}=-\frac{1}{2} \sum_{i, j}\left[\left(\overline{v_{\mathrm{L}}}, \overline{v_{\mathrm{R}}^{c}}\right)_{i} M(h, s)_{i j}\left(\begin{array}{c}
v_{\mathrm{L}}^{c} \\
v_{\mathrm{R}}
\end{array}\right)_{j}+\text { h.c. }\right]
$$

where

$$
M(h, s)_{i j}=\left(\begin{array}{cc}
0 & m_{\mathrm{D}}\left(1+\frac{h}{v}\right) \\
m_{\mathrm{D}}\left(1+\frac{h}{v}\right) & M_{\mathrm{M}}\left(1+\frac{s}{w}\right)
\end{array}\right)_{i j},
$$

with complex $m_{\mathrm{D}}$ and real $M_{\mathrm{M}}$ being symmetric $3 \times 3$ matrices, so $M(0,0)$ is a complex symmetric $6 \times 6$ matrix. The propagating states, obtained by the diagonalization of the matrix $M(0,0)$, will be mixtures of the left- and right-handed neutrinos with masses $m_{i}$ for the former and $M_{j}$ for the latter. It is natural to assume the hierarchy $m_{i} \ll M_{j}$ when the heavy states can be integrated out and we obtain an effective operator with Majorana mass terms for the left-handed neutrinos

$$
\mathscr{L}_{\mathrm{dim}-5}^{v}=-\frac{1}{2} \sum_{i} m_{\mathrm{M}, i}\left(1+\frac{h}{v}\right)^{2}\left(\overline{v_{i, \mathrm{~L}}^{\prime} c} v_{i, \mathrm{~L}}^{\prime}+\text { h.c. }\right) \text {. }
$$

The Majorana masses $m_{\mathrm{M}, i}=m_{i}^{2} / M_{i}$ can be of $\mathrm{O}(100 \mathrm{meV})$ if $m_{i} \sim \mathrm{O}(100 \mathrm{keV}$ ) (as for the charged leptons) and the masses of the right-handed neutrinos are around $\mathrm{O}(100 \mathrm{GeV})$.

Similarly to the standard model, the neutral gauge fields mix. The mixing is described by the $3 \times 3$ orthogonal matrix $O\left(\sin \theta_{\mathrm{W}}, \cos \theta_{T}\right)$,

$$
\left(\begin{array}{c}
W_{\mu}^{3} \\
B_{\mu}^{\prime} \\
Z_{\mu}^{\prime}
\end{array}\right)=O\left(\sin \theta_{\mathrm{W}}, \cos \theta_{T}\right)\left(\begin{array}{c}
Z_{\mu} \\
T_{\mu} \\
A_{\mu}
\end{array}\right),
$$


with $\theta_{\mathrm{W}}$ being the usual weak and $\theta_{T}$ is a new mixing angle. In terms of the new fields the neutral current Lagrangian is the sum of the usual QED term,

$$
\mathscr{L}_{\mathrm{QED}}=-e A_{\mu} J_{\mathrm{em}}^{\mu}, \quad J_{\mathrm{em}}^{\mu}=\sum_{f=1}^{3} \sum_{j=1}^{3} e_{j}\left(\bar{\psi}_{q, j}^{f}(x) \gamma^{\mu} \psi_{q, j}^{f}(x)+\bar{\psi}_{l, j}^{f}(x) \gamma^{\mu} \psi_{l, j}^{f}(x)\right),
$$

$e_{j}$ being the electric charge of the field $\psi_{j}$ in units of the unit charge $e$, and two neutral currents. Of the latter one is coupled to the $\mathrm{Z}^{0}$ boson,

$$
\mathscr{L}_{Z}=-e Z_{\mu}\left(\cos \theta_{T} J_{Z}^{\mu}-\sin \theta_{T} J_{T}^{\mu}\right)=-e Z_{\mu} J_{Z}^{\mu}+\mathrm{O}\left(\theta_{T}\right)
$$

and the other to the $\mathrm{T}^{0}$ boson,

$$
\mathscr{L}_{T}=-e T_{\mu}\left(\sin \theta_{T} J_{Z}^{\mu}+\cos \theta_{T} J_{T}^{\mu}\right) .
$$

$J_{Z}^{\mu}$ is the usual neutral current of the standard model, while the new neutral current has the same dependence on fermion dynamics with different coupling strength:

$$
J_{T}^{\mu}=\sum_{f=1}^{3} \sum_{j=1}^{3} \frac{\gamma_{Z}^{\prime} r_{j}+\gamma_{Z Y}^{\prime} y_{j}}{\sin \theta_{\mathrm{W}}}\left(\bar{\psi}_{q, j}^{f}(x) \gamma^{\mu} \psi_{q, j}^{f}(x)+\bar{\psi}_{l, j}^{f}(x) \gamma^{\mu} \psi_{l, j}^{f}(x)\right) .
$$

\section{Possible consequences and constraints on the parameter space}

Our model may explain potentially the following observations:

- If the lightest new particle is sufficiently stable, then it will be a candidate for WIMP dark matter.

- A possible origin of effective Majorana mass terms and the observed neutrino oscillations for the left-handed neutrinos emerging from the diagonalization of the mass matrix $M(h, s)_{i j}$.

- The diagonalization of the mass matrix $M(h, s)_{i j}$ leads to the appearance of the PontecorvoMaki-Nakagawa-Sakata matrix in the charged current interactions, which can have a CPviolating phase leading to stronger $\mathrm{CP}$ violation in the lepton sector than the similar CabibboKobayashi-Maskawa matrix gives in the quark sector.

- The vacuum of the $\chi$ scalar has a charge $z_{j}=-1$ (or $r_{j}=-1$ ) that may be a source of the current accelerated expansion of the universe.

- The second scalar together with the established BEH field can cause hybrid inflation.

The credibility requirement for the model is to answer the following question: Is there any region of the parameter space of the model that is not excluded by experimental results, both established in standard model phenomenology and elsewhere? We shall address this question in detail as well as studies of the possible consequences listed above in subsequent publications. Here we mention that the study of the ultraviolet behaviour of the model shows that the couplings of the scalar sector and the largest Yukawa coupling of the right handed neutrinos can be constrained by requiring a stable scalar potential and the perturbative value of its couplings up to the Planck scale [13]. 


\section{Conclusions}

In this contribution we have proposed the simplest renormalizable and anomaly free extension of the standard model gauge group by a $U(1)_{Z}$ group, assuming the existence of three right-handed neutrinos and a complex scalar that are neutral with respect to the standard model interactions. In order to fix the new charges of the particle spectrum we assumed that the left- and righthanded neutrinos have opposite $Z$-charges. This model predicts the existence of three massive right-handed neutrinos, a massive scalar particle and a massive neutral vector boson. The gauge invariant Yukawa terms of the neutrinos can provide a field theoretical basis to predict effective Majorana masses for the propagating mass eigenstates of the standard neutrinos and also to explain neutrino oscillations.

In order that the predictions of the model be credible, we have to answer whether there is any region of the parameter space that is not excluded by experimental results established in standard model phenomenology or elsewhere. To answer such a question with satisfaction, extensive studies are needed, which forecasts an exciting research project. An analysis of the ultraviolet behaviour of the scalar couplings is presented in Ref. [13].

\section{References}

[1] G. Pasztor in these proceedings.

[2] A. Sopczak in these proceedings.

[3] J. Brodzicka in these proceedings.

[4] O. V. Boeriu in these proceedings.

[5] Particle Data Group Collaboration, M. Tanabashi et. al., Review of Particle Physics, Phys. Rev. D98 (2018), no. 3030001.

[6] F. Bezrukov and M. Shaposhnikov, Standard Model Higgs boson mass from inflation: Two loop analysis, JHEP 07 (2009) 089 [0 904 . 1537].

[7] G. Degrassi, S. Di Vita, J. Elias-Miro, J. R. Espinosa, G. F. Giudice, G. Isidori and A. Strumia, Higgs mass and vacuum stability in the Standard Model at NNLO, JHEP 08 (2012) 098 [1205. 6497].

[8] S. Weinberg, A Model of Leptons, Phys. Rev. Lett. 19 (1967) 1264-1266.

[9] F. Englert and R. Brout, Broken Symmetry and the Mass of Gauge Vector Mesons, Phys. Rev. Lett. 13 (1964) 321-323. [,157(1964)].

[10] P. W. Higgs, Broken Symmetries and the Masses of Gauge Bosons, Phys. Rev. Lett. 13 (1964) 508-509. [,160(1964)].

[11] Z. Trócsányi, Super-weak force and neutrino masses, 1812.11189.

[12] T. Appelquist, B. A. Dobrescu and A. R. Hopper, Nonexotic neutral gauge bosons, Phys. Rev. D68 (2003) 035012 [hep-ph/0212073].

[13] Z. Péli and Z. Trócsányi, Stability of the vacuum as constraint on $U(1)$ extensions of the standard model, 1902.02791. 